\title{
NON-PERTURBATIVE MASS AND CHARGE RENORMALIZATION IN RELATIVISTIC NO-PHOTON QUANTUM ELECTRODYNAMICS
}

\author{
CHRISTIAN HAINZL AND HEINZ SIEDENTOP
}

\begin{abstract}
Starting from a formal Hamiltonian as found in the physics literature - omitting photons - we define a renormalized Hamiltonian through charge and mass renormalization. We show that the restriction to the oneelectron subspace is well-defined. Our construction is non-perturbative and does not use a cut-off.

The Hamiltonian is relevant for the description of the Lamb shift in muonic atoms.
\end{abstract}

\section{INTRODUCTION}

According to Dirac's hole theory the vacuum consists of electrons which occupy the negative energy states of the free Dirac operator (Dirac sea). Dirac postulated that their charge is not measurable. However, if one introduces an external electric field, e.g., the field of a nucleus, these electrons should rearrange, occupying the negative energy states of the Dirac operator with the external electric field. Physically speaking, the nucleus polarizes the vaccuum. (This rearrangement may be interpreted as the creation of virtual electron-positron pairs when expressed in terms of the free Dirac operator.) In other words, the vacuum is polarized. Dirac 2 ] indicates that these polarization effects result in a logarithmically divergent charge density, which cannot be neglected. As a solution, he suggested that a momentum cut-off must be introduced, since he expected that the Dirac equation would fail for energies higher than $137 m c^{2}$. In 3] he changed this train of thought and suggested that the infinities occurring should be absorbed by a procedure which is now called charge renormalization. A similar step was independently undertaken by Furry and Oppenheimer in [8] who circumvented the hole theory by introducing annihilation and creation operators.

Heisenberg [11] clarified Dirac's picture and generalized his approach extracting the physically relevant terms by subtraction of an unambiguous infinite constant, at least to first order in $\alpha$. Serber [26] and Uehling [29] gave detailed calculations (in first order of $\alpha$ ). Uehling demonstrated that the vacuum polarization alters the

Date: March 17, 2003.

Key words and phrases. quantum electrodynamics, vacuum polarization, mass renormalization, charge renormalization.

(C) 2003 The authors. Reproduction of this article for non-commercial purposes by any means is permitted.

C.H. has been supported by a Marie Curie Fellowship of the European Community program "Improving Human Research Potential and the Socio-economic Knowledge Base" under contract number HPMFCT-2000-00660. Both authors acknowledge partial support through the European Union's IHP network Analysis \& Quantum HPRN-CT-2002-00277. 
Coulomb potential of a charged particle resulting in the electron being slightly more bound in the $s$-states (angular momentum 0) of hydrogenic atoms. Later Weisskopf 31] gave a thorough discussion of the physics involved in charge renormalization.

After the experiments of Lamb and Retherford [18] in 1947, which gave a much higher discrepancy concerning the hyperfine structure of hydrogen, in addition to a different sign, than Uehling's calculation showed, and the first explanation by Bethe [1, the insight into quantum electrodynamics (QED) changed and the interaction with the radiation field turned out to be the dominating part in describing the splitting of the energy levels of hydrogenic atoms beyond the Dirac equation. Similar to vacuum polarization, which was now treated together with the radiative corrections, the photon interaction caused fundamental problems such as infinities, which were "removed" - at least in first order of $\alpha$ - by mass renormalization by Tomonaga, Schwinger, and Feynman. Eventually, Dyson "succeeded" with the renormalization program to every order in $\alpha$. Since then, QED has proven to be of extraordinary predictive power. (We refer the reader interested in more historical details to Schweber [24].)

But despite the predictive power of quantum electrodynamics, the description in terms of perturbation theory causes great uneasiness among mathematicians; a mathematically consistent formulation of QED is still unknown; in fact Dyson [5] indicated that the perturbation theory is divergent. A self-adjoint Hamiltonian for QED is not known.

In the present paper we address a particular kind of singularities arising in QED, namely those stemming from the vacuum polarization. As opposed to the prevalent physics literature we will not use any Feynman diagram but will rather construct a Hamiltonian (in Coulomb gauge). This we have in common with the above cited early works in the field. However, the fact that we start from a formal Hamiltonian and renormalize it non-perturbatively distinguishes us from those authors.

Altough our approach is rigorous, the resulting renormalization is far from being of academic interest only. In fact, the restriction $D_{\text {ren }}$ of the fully renormalized Hamiltonian $\mathbb{H}$ to the one-particle electron sector accounts already for a precise description of the low energy levels of $\mu$-mesonic atoms where the vacuum polarization effect dominates the radiative corrections by far, since the Bohr orbits traverse the support of the polarization potential in this case. (See, e.g., Peterman and Yamaguchi [21, Glauber et al. 9], Milonni [19], Weinberg [30], and Greiner et al [10.)

\section{MOdeL}

In relativistic QED the quantized electron-positron field $\Psi(x)$, which is an operator valued spinor, is written formally as

$$
\Psi(x)=a(x)+b^{*}(x)
$$

where $a(x)$ annihilates an electron at $x$ and $b^{*}(x)$ creates a positron at $x$. (We use the notation that $x=(\mathbf{x}, \sigma) \in \Gamma=\mathbb{R}^{3} \times\{1,2,3,4\}$, where $\sigma$ is the spin index and $\int d x$ denotes integration over $\mathbb{R}^{3}$ and a summation over $\sigma$.) The underlying Hilbert space is given by $\mathfrak{H}=L^{2}(\Gamma)$. 
The definition of a one-electron, respectively one-positron, state will correspond to the positive, respectively negative, energy solutions of the Dirac operator

$$
D^{\varphi}=\boldsymbol{\alpha} \cdot \frac{1}{i} \nabla+\beta-\alpha \varphi
$$

in which $\boldsymbol{\alpha}, \beta$ denote the $4 \times 4$ Dirac matrices. The constant $\alpha$ is a positive real number, the Sommerfeld fine structure constant which is approximately $1 / 137$. (We have picked units in which the electron mass is equal to one.)

We will not assume that the nucleus is a point particle; we rather associate with it a density $n \in L^{1}(\mathbb{R})$ whose integral gives the atomic $Z$ number of the atom under consideration. For technical convenience we assume $n$ to be a spherically symmetric Schwartz function whose Fourier transform has compact support.

We remark: it is an experimental fact that the nucleus is not a point particle but an extended object. In fact the numerical calculations of the Lamb shift depend on the size of the support, which actually limits the accuracy of numerical value of the calculation of the Lamb shift because of the experimental uncertainty of the radius of the nucleus (Weinberg [30, p. 593). (A point nucleus leads also to mathematical difficulties, since the renormalized potential is more singular than the Coulomb potential, i.e., it could not be controlled by the kinetic energy (see Uehling [29] and Subsection 3.5 of this paper).

The electric potential of the nucleus is given as

$$
\varphi=|\cdot|^{-1} * n .
$$

An application of the Young inequality shows that the nuclear potential

$$
\varphi \in L^{3+\epsilon}\left(\mathbb{R}^{3}\right) \cap L^{\infty}\left(\mathbb{R}^{3}\right)
$$

under our assumption on the nuclear density $n$ for any positive $\epsilon$. Moreover, by Newton's theorem

$$
0 \leq \varphi(\mathbf{x}) \leq Z /|\mathbf{x}| .
$$

For completeness we note the following fact whose proof is obvious:

Lemma 1. Fix $\alpha Z \in[0,1)$ and assume $n$ to be a non-negative Schwartz function with $\int n=Z$ and $\varphi=|\cdot|^{-1} * n$. Then, $D^{\varphi}$ is selfadjoint with domain $H^{1}(\Gamma)$, i.e., the same domain as the free Dirac operator $D_{0}$. Moreover, if $n$ is spherically symmetric, $D^{\varphi}$ has no negative discrete spectrum and its $k$-th eigenvalues is bounded from below by the $k$-th eigenvalue of the Coulomb Dirac operator $D^{Z /|\cdot|}$.

We remark that Lemma 1 implies that the lowest eigenvalue is positive.

Now, we can specify the electron and positron state spaces $\mathfrak{H}_{+}^{\varphi}$ and $\mathcal{C H}_{-}^{\varphi}$ respectively: the orthogonal projection on $\mathfrak{H}_{+}^{\varphi}$ and $\mathcal{C H}_{-}^{\varphi}$ are defined as $P_{+}^{\varphi}:=\chi_{[0, \infty)}\left(D_{\alpha, m}^{\varphi}\right)$ and $P_{-}^{\varphi}:=1-P_{+}^{\varphi}$. The (anti-unitary) charge conjugation operator is given on $\mathfrak{H}$ by $(\mathcal{C} \psi)(\mathbf{x})=i \beta \alpha_{2} \overline{\psi(\mathbf{x})}$. In momentum space it acts as $(\hat{\mathcal{C}} \hat{\psi})(\mathbf{p})=i \beta \alpha_{2} \overline{\hat{\psi}(-\mathbf{p})}$. (Here and in the following we follow the notation of Thaller [28; see also 12.)

Note that the Hilbert space can be written as the orthogonal sum

$$
\mathfrak{H}=\mathfrak{H}_{+}^{\varphi} \oplus \mathfrak{H}_{-}^{\varphi} .
$$

Correspondingly $a^{*}(f)$ creates an electron in the state $P_{+}^{\varphi} f$, whereas $b^{*}(g)$ creates a positron with wave function $\mathcal{C} P_{-}^{\varphi} g$. Note that the definition of the operator $a$ and $b$ depends explicitly on the choice of the potential $\varphi$. 
The Hamiltonian for the non-interacting electron-positron field is given by

$$
\mathbb{D}^{\varphi}=\int d x: \Psi^{*}(x) D^{\varphi} \Psi(x):
$$

where : : denotes normal ordering, i.e., anti-commuting of all creation operators to the left of all annihilations operators ignoring the anti-commutators.

Note, that for our renormalization procedure the choice of the electron-positron subspaces as the positive and negative eigenspaces of $D^{\varphi}$ is crucial, in fact it is a choice already proposed by Dirac [2].

The creation and annihilation operators fulfill the canonical anti-commutation relations

$$
\begin{aligned}
\{a(f), a(g)\} & =\left\{a^{*}(f), a^{*}(g)\right\}=\{a(f), b(g)\}=\left\{a^{*}(f), b^{*}(g)\right\} \\
& =\left\{a^{*}(f), b(g)\right\}=\left\{a(f), b^{*}(g)\right\}=0,
\end{aligned}
$$

and

$$
\left\{a(f), a^{*}(g)\right\}=\left(f, P_{+}^{\varphi} g\right),\left\{b^{*}(f), b(g)\right\}=\left(f, P_{-}^{\varphi} g\right) .
$$

Formally this is equivalent to

$$
\begin{aligned}
\{a(x), a(y)\} & =\left\{a^{*}(x), a^{*}(y)\right\}=\{a(x), b(y)\}=\left\{a^{*}(x), b^{*}(y)\right\} \\
& =\left\{a^{*}(x), b(y)\right\}=\left\{a(x), b^{*}(y)\right\}=0,
\end{aligned}
$$

and

$$
\left\{a(x), a^{*}(y)\right\}=P_{+}^{\varphi}(x, y),\left\{b^{*}(x), b(y)\right\}=P_{-}^{\varphi}(x, y)
$$

where $P_{+}^{\varphi}(x, y), P_{-}^{\varphi}(x, y)$ are the integral kernels of the projectors $P_{+}^{\varphi}, P_{-}^{\varphi}$.

If there is no external potential there should be no polarization effects present. It is therefore expected that the difference $Q^{\varphi}$ of one-particle density matrices of the perturbed and unperturbed vacua

$$
Q^{\varphi}:=P_{+}^{\varphi}-P_{+}^{0}=-P_{-}^{\varphi}+P_{-}^{0}
$$

plays a central role in defining the renormalized Hamiltonian. Using Cauchy's formula we can express the $Q^{\varphi}$ in terms of the respective resolvents (Kato [14, Section VI,5, Lemma 5.6)

$$
Q^{\varphi}=\frac{1}{2 \pi} \int_{-\infty}^{\infty} d \eta\left(\frac{1}{D^{\varphi}+i \eta}-\frac{1}{D^{0}+i \eta}\right)
$$

The difference of $Q^{\varphi}$ and of the first order resolvent expansion, i.e.,

$$
Q^{\varphi}-\frac{\alpha}{2 \pi} \int_{-\infty}^{\infty} d \eta \frac{1}{D^{0}+i \eta} \varphi \frac{1}{D^{0}+i \eta}
$$

will contribute to the renormalized operator as follows: one interprets its spin summed diagonal as density. The corresponding electric potential should be added to the one particle operator. To avoid any unnecessary difficulties defining the operator we split (14) again in three summands motivated by iterating the resolvent equation:

$$
\alpha^{2} Q_{2}+\alpha^{3} Q_{3}+\alpha^{4} Q_{4},
$$


where the indices $2,3,4$ indicate the number of $\varphi$ 's in the expression, i.e.,

$$
\begin{aligned}
Q_{2} & :=\frac{1}{2 \pi} \int_{-\infty}^{\infty} d \eta \frac{1}{D^{0}+i \eta} \varphi \frac{1}{D^{0}+i \eta} \varphi \frac{1}{D^{0}+i \eta} \\
Q_{3} & :=\frac{1}{2 \pi} \int_{-\infty}^{\infty} d \eta \frac{1}{D^{0}+i \eta} \varphi \frac{1}{D^{0}+i \eta} \varphi \frac{1}{D^{0}+i \eta} \varphi \frac{1}{D^{0}+i \eta}, \\
Q_{4} & :=\frac{1}{2 \pi} \int_{-\infty}^{\infty} d \eta \frac{1}{D^{0}+i \eta} \varphi \frac{1}{D^{0}+i \eta} \varphi \frac{1}{D^{\varphi}+i \eta} \varphi \frac{1}{D^{0}+i \eta} \varphi \frac{1}{D^{0}+i \eta} .
\end{aligned}
$$

We can immediately remark that the density corresponding to $Q_{2}$ vanishes: the terms linear in the Dirac matrices vanish after summation over $\sigma$, since the Dirac matrices are traceless; the remaining terms are odd in $\eta$ and vanish after integration over $\eta$. Consequently, we can disregard this term in defining the operator.

We now define the density

$$
\rho_{3}(\mathbf{x}):=(2 \pi)^{-3} \int_{\mathbb{R}^{3}} d \mathbf{p} \int_{\mathbb{R}^{3}} d \mathbf{q} \sum_{\sigma=1}^{4} e^{i(\mathbf{p}-\mathbf{q}) \cdot \mathbf{x}} \hat{Q}_{3}(\mathbf{p}, \sigma ; \mathbf{q}, \sigma)
$$

where

$$
\begin{aligned}
\hat{Q}_{3}(\mathbf{p}, \mathbf{q})=\frac{1}{2 \pi} \int_{-\infty}^{\infty} d \eta & \int_{\mathbb{R}^{3}} d \mathbf{p}_{1} \int_{\mathbb{R}^{3}} d \mathbf{p}_{2}\left(D_{\mathbf{p}}+i \eta\right)^{-1} \circ \hat{\varphi}\left(\mathbf{p}-\mathbf{p}_{1}\right) \circ\left(D_{\mathbf{p}_{1}}+i \eta\right)^{-1} \\
& \circ \hat{\varphi}\left(\mathbf{p}_{1}-\mathbf{p}_{2}\right) \circ\left(D_{\mathbf{p}_{2}}+i \eta\right)^{-1} \circ \hat{\varphi}\left(\mathbf{p}_{2}-\mathbf{q}\right) \circ\left(D_{\mathbf{q}}+i \eta\right)^{-1}
\end{aligned}
$$

with $D_{\mathbf{r}}:=\boldsymbol{\alpha} \cdot \mathbf{r}+\beta$. The corresponding electric potential is

$$
P_{3}:=\rho_{3} *|\cdot|^{-1} \text {. }
$$

The quadratic form defining $P_{4}$ is given by

$$
\left(\psi, P_{4} \psi\right):=\operatorname{tr}\left(\chi Q_{4}\right)
$$

where $\chi(\mathbf{x}):=\int d y|\psi(y)|^{2} /|\mathbf{y}-\mathbf{x}|$.

It will be useful to introduce the function $C$

$$
\begin{aligned}
C(\mathbf{k}) & =\frac{1}{2} \mathbf{k}^{2} \int_{0}^{1} d x\left(1-x^{2}\right) \log \left[1+\mathbf{k}^{2}\left(1-x^{2}\right) / 4\right] \\
& =\frac{1}{3} \mathbf{k}^{2}\left[\left(1-\frac{2}{\mathbf{k}^{2}}\right) \sqrt{1+\frac{4}{\mathbf{k}^{2}}} \log \frac{\sqrt{1+4 / \mathbf{k}^{2}}+1}{\sqrt{1+4 / \mathbf{k}^{2}}-1}+\frac{4}{\mathbf{k}^{2}}-\frac{5}{3}\right]
\end{aligned}
$$

as already done by Serber [26] and Uehling [29] and later by Pauli and Rose [20, Jauch and Rohrlich [13, Schwinger [25, and Klaus and Scharf [16. The vacuum polarization potential $U$, also known as Uehling potential, is defined via its Fourier transform

$$
\hat{U}(\mathbf{k})=\hat{\varphi}(\mathbf{k}) \frac{C(\mathbf{k})}{\pi|\mathbf{k}|^{2}} .
$$

The renormalized one-particle operator is

$$
D_{\text {ren }}:=\boldsymbol{\alpha} \cdot \frac{1}{i} \nabla+\beta-\alpha \varphi-\alpha^{2} U+\alpha^{2} X-\alpha^{4} P_{3}-\alpha^{5} P_{4}
$$

where $X$ is the renormalized operator with integral kernel

$$
\alpha X(x, y):=\frac{Q^{\varphi}(x, y)}{|\mathbf{x}-\mathbf{y}|} .
$$


To introduce the operator $D_{\text {ren }}$ might appear unmotivated at this point. However, it has a solid physical motivation: it emerges through mass and charge renormalization from the canonical formal text book Hamiltonian (see, e.g., Milonni [19], p. 385, Formula (11.25)) for the interaction of electrons when there are no photons present. In turn the charge and mass renormalization originates in three physical principles $\mathbf{W} \mathbf{1}, \mathbf{W} \mathbf{2}$, and $\mathbf{W} \mathbf{3}$ as we will explain in Section 3

Moreover, and this is our main mathematical result, the operator $P_{+}^{\varphi} D_{\text {ren }}$ on $\mathfrak{H}_{+}^{\varphi}$ turns out to be well defined and self-adjoint on the same form domain as the free Dirac operator:

Theorem 1. Assume $\alpha Z \in[0,1)$ and $\varphi=n *|\cdot|^{-1}$ with spherically symmetric Schwartz function with compact support in Fourier space. Then the quadratic forms $(\psi, U \psi),(\psi, X \psi),\left(\psi, P_{3} \psi\right),\left(\psi, P_{4} \psi\right)$ on $P_{+}^{\varphi}\left(H^{1}(\Gamma)\right)$ are relatively form bounded with respect to $\left(\psi,\left|D^{0}\right| \psi\right)$ with form bound zero.

This has the following consequence:

Corollary 1. There exists a unique self-adjoint operator $D_{+}$fulfilling $\left(\psi, D_{+} \psi\right)=$ $\left(\psi,\left(D^{\varphi}+\alpha^{2} X-\alpha^{2} U-\alpha^{4} P_{3}-\alpha^{5} P_{4}\right) \psi\right)$ for all $\psi \in P_{+}^{\varphi}\left(H^{1}(\Gamma)\right)$ with form domain $P_{+}^{\varphi}\left(H^{1 / 2}(\Gamma)\right)$. Furthermore it is bounded from below.

Proof. Define $c:=\sqrt{2}$ and $M:=\sqrt{2}\|\varphi\|_{\infty}$. Then, obviously $0 \leq\left(c^{2}-2\right)\left(D^{0}\right)^{2}-$ $2 \varphi^{2}+M^{2}$ implying $\left(D^{0}\right)^{2} \leq c^{2}\left(D_{0}^{2}-2 \Re\left(\varphi D^{0}\right)+\varphi^{2}\right)+M^{2}+2 M c\left|D^{\varphi}\right|$. Rewriting this and taking the square root - which is an operator montone function - yields

$$
\left|D^{0}\right| \leq c\left|D^{\varphi}\right|+M
$$

This means that infinitesimal form boundedness with respect to $\left|D^{0}\right|$ implies also infinitesimal form boundedness with respect to $\left|D^{\varphi}\right|$. Moreover, we have $\left(\psi,\left|D^{\varphi}\right| \psi\right)=$ $\left(\psi, D^{\varphi} \psi\right)$ for $\psi \in P_{+}^{\varphi}\left(H^{1}(\Gamma)\right)$.

Thus, according to the KLMN theorem (Reed and Simon [23], Theorem X.17) there exists a unique self-adjoint operator $D_{+}$on $\mathfrak{H}_{+}^{\varphi}$ whose form domain is the form domain of $D^{\varphi}$ (which - since $\varphi$ is bounded - is the form domain of $P_{+}^{\varphi} D^{0}$, i.e., $\left.P_{+}^{\varphi} H^{1 / 2}(\Gamma)\right)$. Moreover, the quadratic form of $D_{+}$fulfills

$$
\left(\psi, D_{+} \psi\right)=\left(\psi,\left(D^{\varphi}+\alpha^{2} X-\alpha^{2} U-\alpha^{4} P_{3}-\alpha^{5} P_{4}\right) \psi\right) .
$$

As already mentioned in the introduction, the Hamiltonian (23) can be used to describe $\mu$-mesonic atoms where the interaction with the photon field is negligible as indicated experimentally by Peterman and Yamaguchi [21] and theoretically by Glauber et al 9 .

\section{Physical "Derivation" of the Renormalized Hamiltonian}

We start with the formal expression for the interaction of electrons when no photons are present (Kroll and Lamb [17, French and Weisskopf 7]) found also in text books (see, e.g., Milonni [19]):

$$
\mathbb{W}_{\mathrm{ur}}=\frac{1}{2} \int d x \int d y \frac{\Psi^{*}(x) \Psi(x) \Psi^{*}(y) \Psi(y)}{|\mathbf{x}-\mathbf{y}|} .
$$

The Hamiltonian describing our system is formally given by

$$
\mathbb{H}_{\mathrm{ur}}=\mathbb{D}_{\mathrm{ur}}^{\varphi}+\alpha \mathbb{W}_{\mathrm{ur}}
$$


where

$$
\mathbb{D}_{\mathrm{ur}}^{\varphi}:=\int d x \Psi^{*}(x) D^{\varphi} \Psi(x) .
$$

It is well known that this expression contains several singular terms. In particular it does not even contain any normal ordering. The remaining part of this section can be viewed as manipulating on it a physical allowed way and transforming it to a physically equivalent expression that is mathematically meaningful, namely the renormalized Hamiltonian. We emphasize that none of the steps taken is mathematically justified, i.e., the eventual justification of the renormalized Hamiltonian is its successful predictive power.

We use three guiding principles to transform expressions for the energy into other physically equivalent ones as formulated and justified by Weisskopf [31, p. 6: “The following three properties of the vacuum electrons are assumed to be irrelevant:

W1: The energy of the vacuum electrons in field free space.

W2: The charge and current density of the vacuum electrons in field free space.

W3: A field independent electric and magnetic polarizability that is constant in space and time."

Similar procedures have been suggested by Heisenberg [1], French and Weisskopf 7], Kroll and Lamb [1], and Dyson 4].

Exploiting the canonical anti-commutation relations (10) we can rewrite (28). For the one-particle part we have

$$
\mathbb{D}_{\mathrm{ur}}^{\varphi}=\mathbb{D}^{\varphi}+\int d x\left(D^{\varphi} P_{-}^{\varphi}\right)(x, x) .
$$

The last summand is a - although infinite - constant which we drop, since it does not influence energy differences. For the two-particle part we get

$$
\begin{aligned}
\mathbb{W}_{\mathrm{ur}}= & \frac{1}{2} \int d x \int d y \frac{: \Psi^{*}(x) \Psi(x) \Psi^{*}(y) \Psi(y):}{|\mathbf{x}-\mathbf{y}|} \\
& +\frac{1}{2} \int d x \int d y: \Psi^{*}(x) \Psi(y): \frac{P_{+}^{\varphi}(x, y)-P_{-}^{\varphi}(x, y)}{|\mathbf{x}-\mathbf{y}|} \\
& +\int d x \int d y: \Psi^{*}(x) \Psi(x): \frac{P_{-}^{\varphi}(y, y)}{|\mathbf{x}-\mathbf{y}|} \\
& +\frac{1}{2} \int d x \int d y \frac{P_{+}^{\varphi}(x, y) P_{-}^{\varphi}(x, y)}{|\mathbf{x}-\mathbf{y}|}+\frac{1}{2} \int d x \int d y \frac{P_{-}^{\varphi}(x, x) P_{-}^{\varphi}(y, y)}{|\mathbf{x}-\mathbf{y}|} .
\end{aligned}
$$

The last two terms are again constants which we drop. The first term is the normal ordered two-particle interaction which has finite expectation in states of finite kinetic energy. We will denote it by

$$
\mathbb{W}=\frac{1}{2} \int d x \int d y \frac{: \Psi^{*}(x) \Psi(x) \Psi^{*}(y) \Psi(y):}{|\mathbf{x}-\mathbf{y}|} .
$$

The remaining two other terms are one-particle operators of particular interest to us.

Both terms, the classical electrostatic interaction energy of the electron with the polarized Dirac sea called the "non-exchange energy"

$$
\mathbb{P}_{\text {ur }}:=-\int d x \int d y: \Psi^{*}(x) \Psi(x): \frac{P_{-}^{\varphi}(y, y)}{|\mathbf{x}-\mathbf{y}|},
$$


and the exchange energy

$$
\mathbb{X}_{\mathrm{ur}}:=\frac{1}{2} \int d x \int d y: \Psi^{*}(x) \Psi(y): \frac{P_{+}^{\varphi}(x, y)-P_{-}^{\varphi}(x, y)}{|\mathbf{x}-\mathbf{y}|}
$$

are not well defined. (For curiosity we remark that the latter is logarithmically divergent in $\Lambda$, if one introduces a cut-off by $\left.\Psi(x)=\int_{|\mathbf{p}| \leq \Lambda} \hat{\Psi}(\mathbf{p}, \sigma) e^{-i \mathbf{p} \cdot \mathbf{x}} d \mathbf{p}\right)$.

To renormalize the exchange energy we introduce the operators $P_{+}^{0}, P_{-}^{0}$ which are the projectors on the positive and negative subspace of the free Dirac operator $D^{0}$. (Note that we can interpret $P_{-}^{0}$ as the one-particle density matrix of the free Dirac sea.)

3.1. The Renormalization of the Exchange Energy. To renormalize $\mathbb{X}_{\mathrm{ur}}$ we subtract the exchange interaction energy of the electron with the free Dirac sea using Principle W1, i.e.,

$$
\begin{aligned}
\alpha \mathbb{X} & :=\frac{1}{2} \int d x \int d y: \Psi^{*}(x) \Psi(y): \frac{\left[\left(P_{+}^{\varphi}-P_{+}^{0}\right)-\left(P_{-}^{\varphi}-P_{-}^{0}\right)\right](x, y)}{|\mathbf{x}-\mathbf{y}|} \\
& =\alpha \int d x \int d y: \Psi^{*}(x) \Psi(y): X(x, y)
\end{aligned}
$$

with $X$ as defined in (24).

(In physical language this subtraction of an undefined operator - known as "counter term" - is called "mass renormalization". We refer to French and Weisskopf [], Equation (30), for the motivation of this terminology.)

From now on, we will assume that the external potential $\varphi$ is so weak that all there are only positive eigenvalues in the gap of $D^{\varphi}$. Then,

$$
P_{+}^{\varphi}=\frac{1}{2}+\frac{1}{2 \pi} \int_{-\infty}^{\infty} d \eta \frac{1}{D^{\varphi}+i \eta}
$$

as well as

$$
P_{+}^{0}=\frac{1}{2}+\frac{1}{2 \pi} \int_{-\infty}^{\infty} d \eta \frac{1}{D^{0}+i \eta}
$$

(Kato 14, Section VI,5, Lemma 5.6). Thus,

$$
Q^{\varphi}=\frac{\alpha}{2 \pi} \int_{-\infty}^{\infty} d \eta \frac{1}{D^{0}+i \eta} \varphi \frac{1}{D^{0}+i \eta}+\frac{\alpha^{2}}{2 \pi} \int_{-\infty}^{\infty} d \eta \frac{1}{D^{0}+i \eta} \varphi \frac{1}{D^{\varphi}+i \eta} \varphi \frac{1}{D^{0}+i \eta}
$$

where the first summand of the right hand side is denoted by $\alpha Q_{1}$ and the second summand is treated in (14) through (16).

Furthermore, since

$$
\int_{\mathbb{R}^{3}} d \mathbf{p} \frac{\mathbf{p}^{2}|\hat{\varphi}(\mathbf{p})|^{2}}{1+|\mathbf{p}|}<\infty
$$

the potential $\varphi$ is regular in the sense of Klaus and Scharf [15], namely the operator $Q^{\varphi} \in \mathfrak{S}_{2}(\mathfrak{H})$, i.e., $Q^{\varphi}$ is an Hilbert-Schmidt operator. (See also 12], Theorem 4.) This allows to show the finiteness of the exchange energy between the oneparticle density matrix of the electron-positron field and the difference of the density matrices of the polarized Dirac sea and the free Dirac sea.

To formulate the next lemma we fix the following notation: let $C_{p, q}$ be the optimal constant in the generalized Young inequality, i.e., $\|f * g\|_{r} \leq C_{p, q}\|f\|_{p}\|g\|_{q}$, $1<p, q, r<\infty, r^{-1}+1=p^{-1}+q^{-1}$. 
Lemma 2. Let $\psi \in L^{3}(\Gamma) \cap L^{2}(\Gamma)$. Then

$$
\left|\int d x \int d y \frac{\overline{\psi(x)} Q^{\varphi}(x, y) \psi(y)}{|\mathbf{x}-\mathbf{y}|}\right| \leq \sqrt{C_{3 / 2,3 / 2}\left\|1 /|\cdot|^{2}\right\|_{3 / 2, w}}\left\|Q^{\varphi}\right\|_{2}\|\psi\|_{3}^{2},
$$

and for every $\epsilon>0$ there exists a constant $C_{\epsilon}>0$ such that

$$
\left|\int d x \int d y \frac{\overline{\psi(x)} Q^{\varphi}(x, y) \psi(y)}{|\mathbf{x}-\mathbf{y}|}\right| \leq \epsilon\|\psi\|_{3}^{2}+C_{\epsilon}\|\psi\|_{2}^{2} .
$$

Proof. Since $Q^{\varphi}$ is a Hilbert-Schmidt operator we get using the Schwarz inequality

$$
\begin{aligned}
L:=\mid \int d x & \int d y \frac{\overline{\psi(x)} Q^{\varphi}(x, y) \psi(y)}{|\mathbf{x}-\mathbf{y}|} \mid \\
& \leq\left(\int d x \int d y \frac{|\psi(x)|^{2}|\psi(y)|^{2}}{|\mathbf{x}-\mathbf{y}|^{2}}\right)^{1 / 2}\left(\int d x \int d y\left|Q^{\varphi}(x, y)\right|^{2}\right)^{1 / 2}
\end{aligned}
$$

The second factor of the right hand side is the Hilbert-Schmidt norm $\left\|Q^{\varphi}\right\|_{2}$ of $Q^{\varphi}$.

To estimate the first factor we decompose the kernel into two functions $f(\mathbf{x}):=$ $\chi_{B_{R}(0)}(\mathbf{x}) /|\mathbf{x}|^{2}$ and the rest $g$, i.e., $1 /|\mathbf{x}|^{2}=f(\mathbf{x})+g(\mathbf{x})$.

Thus, using inequality (41) we get

$$
L \leq\left[\left(|\psi|^{2} * f,|\psi|^{2}\right)^{1 / 2}+\left(|\psi|^{2} * g,|\psi|^{2}\right)^{1 / 2}\right]\left\|Q^{\varphi}\right\|_{2} .
$$

We estimate the first and second summand of the first factor on the right hand side separately from above.

The first summand yields using the Hölder inequality followed by the generalized Young inequality (see, e.g., Reed and Simon [22, p. 32)

$$
\left(|\psi|^{2} * f,|\psi|^{2}\right) \leq C_{3 / 2,3 / 2}\left\|\psi^{2}\right\|_{3 / 2}^{2}\|f\|_{3 / 2, w},
$$

where $w$ indicates the weak-norm.

Picking the radius $R=\infty$, i.e., $g=0$, yields immediately (39).

To prove (40) we also use (43) but pick the radius $R>0$ sufficiently small: in this case we need to bound also the second summand containing $g$; we use again Hölder's inequality now followed by using Young's inequality

$$
\left(|\psi|^{2} * g,|\psi|^{2}\right) \leq\left\|\psi^{2}\right\|_{1}^{2}\|g\|_{\infty} .
$$

Thus, the first factor on the right hand side of (42) is bounded by

$$
\sqrt{C_{3 / 2,3 / 2}}\left\|\psi^{2}\right\|_{3 / 2}\|f\|_{3 / 2, w}^{1 / 2}+\left\|\psi^{2}\right\|_{1}\|g\|_{\infty}^{1 / 2}
$$

Since $\|f\|_{3 / 2, w}$ tends to zero as $R$ tends to zero, the claimed inequality follows.

3.2. Electrostatic Vacuum Polarization Energy (Non-Exchange Energy). In the expression for the electrostatic vacuum polarization energy we replace the density of the polarized sea by the difference of this density and the free Dirac sea 
using Principle W2:

$$
\begin{aligned}
\tilde{\mathbb{P}} & =-\int d x \int d y: \Psi^{*}(x) \Psi(x): \frac{P_{-}^{\varphi}(y, y)-P_{-}^{0}(y, y)}{|\mathbf{x}-\mathbf{y}|} \\
& =\int d x \int d y: \Psi^{*}(x) \Psi(x): \frac{Q^{\varphi}(y, y)}{|\mathbf{x}-\mathbf{y}|} \\
& =\int d x \int_{\mathbb{R}^{3}} d \mathbf{y}: \Psi^{*}(x) \Psi(x): \frac{\operatorname{tr}_{\mathbb{C}^{4}} Q^{\varphi}(\mathbf{y}, \mathbf{y})}{|\mathbf{x}-\mathbf{y}|} .
\end{aligned}
$$

(Here and in the following we will denote by $Q^{\varphi}(\mathbf{x}, \mathbf{y})$ the $4 \times 4$ matrix with entries $\left.\left(Q^{\varphi}(\mathbf{x}, \sigma ; \mathbf{y}, \tau)\right)_{\sigma, \tau=1}^{4}.\right)$ However, the integral kernel of $Q^{\varphi}$ is always singular on the diagonal except for vanishing potential as can be seen from (51) implying that $\tilde{\mathbb{P}}$ is not well defined; one more renormalization is necessary. The question how to extract the physical relevant information from $\operatorname{tr}_{\mathbb{C}^{4}} Q^{\varphi}(\mathbf{y}, \mathbf{y})$ was already asked by Dirac [2] and partially answered by Dirac [3], Heisenberg [11, Serber [26], Uehling 29, Weisskopf 31, Schwinger [25], Dyson [4, Klaus and Scharf 16], and others. The proposed solution amounted to a perturbative renormalization according to Principle W3. - One of our main results is that this renormalization can be done non-perturbatively: subtracting the zeroth order expansion $Q_{1}$ of the difference $Q^{\varphi}$ of $P_{+}^{\varphi}$ and $P_{+}^{0}$ will turn $\operatorname{tr}_{\mathbb{C}^{4}} Q^{\varphi}(\mathbf{y}, \mathbf{y})$ into well defined quantities given in (19) and (201).

Recall that

$$
Q_{1}=\frac{1}{2 \pi} \int_{-\infty}^{\infty} d \eta \frac{1}{D^{0}+i \eta} \varphi \frac{1}{D^{0}+i \eta} .
$$

Thus, in momentum space $Q_{1}$ is given by

$$
\hat{Q}_{1}(\mathbf{p}, \mathbf{q})=(2 \pi)^{-5 / 2} \int_{-\infty}^{\infty} d \eta \frac{\boldsymbol{\alpha} \cdot \mathbf{p}+\beta-i \eta}{\mathbf{p}^{2}+1+\eta^{2}} \hat{\varphi}(\mathbf{p}-\mathbf{q}) \frac{\boldsymbol{\alpha} \cdot \mathbf{q}+\beta-i \eta}{\mathbf{q}^{2}+1+\eta^{2}},
$$

which leads to

$$
\operatorname{tr}_{\mathbb{C}^{4}} \hat{Q}_{1}(\mathbf{p}, \mathbf{q})=2^{-1 / 2} \pi^{-3 / 2} \hat{\varphi}(\mathbf{p}-\mathbf{q}) \frac{\mathbf{p} \cdot \mathbf{q}+1-E(\mathbf{p}) E(\mathbf{q})}{E(\mathbf{p}) E(\mathbf{q})(E(\mathbf{p})+E(\mathbf{q}))}
$$

by a straightforward calculation with $E(\mathbf{p})=\sqrt{\mathbf{p}^{2}+1}$. In configuration space we obtain

$$
\begin{aligned}
\operatorname{tr}_{\mathbb{C}^{4}} Q_{1}(\mathbf{x}, \mathbf{y}) & =(2 \pi)^{-3} \int_{\mathbb{R}^{3}} d \mathbf{r} \int_{\mathbb{R}^{3}} d \mathbf{q} e^{i \mathbf{r} \cdot \mathbf{x}} \operatorname{tr}_{\mathbb{C}^{4}} \hat{Q}_{1}(\mathbf{r}, \mathbf{q}) e^{-i \mathbf{q} \cdot \mathbf{y}} \\
& =(2 \pi)^{-3} \int d \mathbf{p} d \mathbf{k} \operatorname{tr}_{\mathbb{C}^{4}} \hat{Q}_{1}(\mathbf{p}-\mathbf{k} / 2, \mathbf{p}+\mathbf{k} / 2) e^{i \mathbf{p} \cdot(\mathbf{x}-\mathbf{y})} e^{-i \mathbf{k} \cdot(\mathbf{x}+\mathbf{y}) / 2} \\
& =\tilde{Q}\left(\mathbf{x}-\mathbf{y}, \frac{\mathbf{x}+\mathbf{y}}{2}\right)
\end{aligned}
$$

after introducing new variables of integration $\mathbf{r}=\mathbf{p}-\mathbf{k} / 2$ and $\mathbf{q}=\mathbf{p}+\mathbf{k} / 2$. Defining $\boldsymbol{\xi}:=\mathbf{x}-\mathbf{y}$ we remark that the "limits" $\lim _{\mathbf{y} \rightarrow \mathbf{x}} \operatorname{tr}_{\mathbb{C}^{4}} Q_{1}(\mathbf{x}, \mathbf{y})$ and $\lim _{\xi \rightarrow 0} \tilde{Q}(\xi, \mathbf{x})$ are formally the same. The corresponding expression $\tilde{\mathbb{P}}$ in the electrostatic energy (45) becomes formally

$$
\int d x \int d \mathbf{y} \frac{: \Psi^{*}(x) \Psi(x): \operatorname{tr}_{\mathbb{C}^{4}} Q_{1}(\mathbf{y}, \mathbf{y})}{|\mathbf{x}-\mathbf{y}|}=\int d k: \widehat{\Psi^{*} \Psi}:(k) \frac{4 \pi}{\mathbf{k}^{2}} \hat{\tilde{Q}}(0, \mathbf{k}),
$$


where $\hat{\tilde{Q}}(\xi, \cdot)$ is the Fourier transform of $\tilde{Q}$ with respect to the second variable for fixed $\xi \neq 0$, i.e., formally

$$
\begin{aligned}
& \hat{\tilde{Q}}(\xi, \mathbf{k})=(2 \pi)^{-3 / 2} \int_{\mathbb{R}^{3}} d \mathbf{p} \operatorname{tr}_{\mathbb{C}^{4}} \hat{Q}_{1}(\mathbf{p}-\mathbf{k} / 2, \mathbf{p}+\mathbf{k} / 2) e^{i \mathbf{p} \cdot \boldsymbol{\xi}} \\
& =\frac{1}{4 \pi^{3}} \hat{\varphi}(\mathbf{k}) \int_{\mathbb{R}^{3}} d \mathbf{p} \frac{\mathbf{p}^{2}-\mathbf{k}^{2} / 4+1-E(\mathbf{p}-\mathbf{k} / 2) E(\mathbf{p}+\mathbf{k} / 2)}{E(\mathbf{p}-\mathbf{k} / 2) E(\mathbf{p}+\mathbf{k} / 2)(E(\mathbf{p}-\mathbf{k} / 2)+E(\mathbf{p}+\mathbf{k} / 2))} e^{i \mathbf{p} \cdot \boldsymbol{\xi}} .
\end{aligned}
$$

We note that the integral (51) is logarithmically divergent at $\boldsymbol{\xi}=0$ independently of the form of the external potential $\varphi$. This shows - as already remarked above that the limit $\lim _{\mathbf{y} \rightarrow \mathbf{x}} \operatorname{tr}_{\mathbb{C}^{4}} Q^{\varphi}(\mathbf{x}, \mathbf{y})$ only exists, if $\varphi$ vanishes.

The expression $\hat{\tilde{Q}}(\boldsymbol{\xi}, \mathbf{k})$ has been intensively studied in the literature (see, e.g., Heisenberg [11, Serber [26, Pauli and Rose [20, Weisskopf [31, and Klaus and Scharf [16]). We will follow mainly the calculations of Pauli and Rose. (Since their treatment is time-dependent one has to set $k_{0}=0$ to translate to our situation.) According to [20], Equations (5) - (9), we can separate $\hat{\tilde{Q}}$ into two terms

$$
\hat{\tilde{Q}}(\boldsymbol{\xi}, \mathbf{k})=F_{1}(\boldsymbol{\xi}, \mathbf{k})+\hat{\varphi}(\mathbf{k}) \mathbf{k}^{2} F_{0}(\boldsymbol{\xi}),
$$

with

$$
F_{0}(\boldsymbol{\xi})=-\frac{1}{16 \pi^{3}} \int_{\mathbb{R}^{3}} d \mathbf{p}\left(1-\frac{\mathbf{p}^{2} \cos ^{2} \theta}{1+\mathbf{p}^{2}}\right) \frac{e^{i \mathbf{p} \cdot \boldsymbol{\xi}}}{\left(1+\mathbf{p}^{2}\right)^{3 / 2}}
$$

$\theta$ being the angle between $\boldsymbol{\xi}$ and $\mathbf{p}$. With this definition of $F_{0}$ the function $F_{1}$ is finite for $\boldsymbol{\xi}=0$ and has there the value

$$
\begin{aligned}
& \hat{\rho}_{\mathrm{vac}}(\mathbf{k}):=F_{1}(0, \mathbf{k}) \\
= & \frac{\hat{\varphi}(\mathbf{k})}{4 \pi^{3}} \int_{\mathbb{R}^{3}} \frac{\mathbf{p}^{2}-\frac{\mathbf{k}^{2}}{4}+1-E\left(\mathbf{p}-\frac{\mathbf{k}}{2}\right) E\left(\mathbf{p}+\frac{\mathbf{k}}{2}\right)}{E\left(\mathbf{p}-\frac{\mathbf{k}}{2}\right) E\left(\mathbf{p}+\frac{\mathbf{k}}{2}\right)\left(E\left(\mathbf{p}-\frac{\mathbf{k}}{2}\right)+E\left(\mathbf{p}+\frac{\mathbf{k}}{2}\right)\right)}+\mathbf{k}^{2} \frac{\mathbf{p}^{2} \sin ^{2} \theta+1}{4 E(\mathbf{p})^{5}} d \mathbf{p} \\
& =\frac{1}{4 \pi^{2}} \hat{\varphi}(\mathbf{k}) C(\mathbf{k})
\end{aligned}
$$

where $C$ is the function defined in (21). While each of the summands in the latter formula decreases like $|\mathbf{p}|^{-3}$ for large values of $|\mathbf{p}|$ and therefore the corresponding parts of the integral are logarithmically divergent, the difference in the integrand decreases like $|\mathbf{p}|^{-5}$ and is therefore convergent.

Pauli and Rose 20] obtain the following asymptotic behavior for $C$ :

$$
C(\mathbf{k}) / \mathbf{k}^{2}=\left\{\begin{array}{ll}
\frac{1}{15} \mathbf{k}^{2}+o\left(\mathbf{k}^{2}\right) & |\mathbf{k}| \rightarrow 0 \\
\frac{2}{3} \log (|\mathbf{k}|)-\frac{5}{9}+o(1) & |\mathbf{k}| \rightarrow \infty
\end{array} .\right.
$$

We note that the second summand on the right hand side of (52) can be written as $4 \pi \hat{n}(\mathbf{k}) F_{0}(\boldsymbol{\xi})$, i.e., this depends only on the density of the nucleus. This implies that it can be dropped according to $\mathbf{W} 3$. This means that $\rho_{\text {vac }}$ as defined in (54) can be considered as the physically relevant density of the polarized vacuum.

3.3. The Fully Renormalized Hamiltonian. The potential of the vacuum polarization density is

$$
U=|\cdot|^{-1} * \rho_{\mathrm{vac}} ;
$$


the corresponding term of the electrostatic interaction of the electron-positron field with the vacuum reads

$$
\begin{array}{r}
-\alpha^{2} \int d x: \Psi^{*}(x) \Psi(x): U(\mathbf{x})=-\alpha^{2} \int d x: \Psi^{*}(x) \Psi(x):|\cdot|^{-1} * \rho_{\mathrm{vac}}(\mathbf{x}) \\
=-\alpha^{2} \int d k: \widehat{\Psi^{*} \Psi}:(k) \frac{4 \pi}{\mathbf{k}^{2}} \rho_{\mathrm{vac}}(\mathbf{k})
\end{array}
$$

where we used $\mathcal{F}\left(|\cdot|^{-1}\right)=\sqrt{2 / \pi}|\cdot|^{-2}$.

According to (45), (38), (17), and (19) the second quantized renormalized polarization energy becomes

$$
\mathbb{P}=\int d x: \Psi^{*}(x) \Psi(x):\left(U(\mathbf{x})+\alpha^{2} P_{3}(\mathbf{x})+\alpha^{3} P_{4}(\mathbf{x})\right) .
$$

Consequently our fully renormalized Hamiltonian is

$$
\mathbb{H}=\mathbb{D}^{\varphi}+\alpha \mathbb{W}+\alpha^{2}(-\mathbb{P}+\mathbb{X}) .
$$

3.4. Physical Interpretation of the Renormalization Procedure of the Vacuum Polarization. In physics literature the subtraction of the singular part of the diagonal term of the one-particle density matrix of the Dirac sea, i.e., the dropping of the second summand (52)) is called charge renormalization for the following reason: the term subtracted from $\tilde{\mathbb{P}}$ to obtain $\mathbb{P}$ is

$$
\alpha^{2} 4 \pi F_{0}(\boldsymbol{\xi}) \int d x: \Psi^{*}(x) \Psi(x): \varphi(\mathbf{x}) .
$$

Formally this result can also be obtained by replacing the square of the (bare) charge $e^{2}=\alpha$ in $D^{\varphi}$

$$
e^{2} \mapsto e^{2}\left(1-4 \pi e^{2} F_{0}(\boldsymbol{\xi})\right) .
$$

Note, that

$$
F_{0}(\boldsymbol{\xi})=-\log (|\boldsymbol{\xi}|)+o(1)
$$

for small $|\boldsymbol{\xi}|$ which leads to a well known formula in the literature (see, e.g., Milonni 19, p. 417). It is interesting that a change in the effective charge due to the polarization of the vacuum was already suggested by Furry and Oppenheimer 8 .

The word polarization is due to the following picture: according to Dirac the electrostatic field causes a redistribution of charge in the Dirac sea, i.e., it polarizes the vacuum. In particular the nucleus polarizes the vacuum in its vicinity causing a screening lowering the effective charge for an observer at a distance.

Another reason for the fact that the infinity of the diagonal part of the density matrix of the Dirac sea in (52) is invisible in experiments is the following: in first order the factor in front of $F_{0}(0)$ changes $\alpha$ in $D^{\varphi}$ by an (infinite) constant only, which does not effect the degeneracy of the eigenvalues of $D^{\varphi}$, i.e., it does not cause a splitting of degenerated eigenvalues, a fact that is confirmed by experiment.

3.5. The Vacuum Polarization Potential of the Coulomb Potential. Recall that the nuclear potential is $\varphi=|\cdot|^{-1} * n$; consequently $\hat{\varphi}(\mathbf{k})=4 \pi \hat{n}(\mathbf{k}) / \mathbf{k}^{2}$. Thus the Fourier transform of the vacuum polarization potential using (54) gives

$$
\hat{U}(\mathbf{k})=\frac{\hat{\varphi}(\mathbf{k}) C(\mathbf{k})}{\pi|\mathbf{k}|^{2}}=4 \frac{\hat{n}(\mathbf{k}) C(\mathbf{k})}{|\mathbf{k}|^{4}}
$$


which is spherically symmetric and compactly supported under our assumptions on the charge distributions of the nucleus.

If we assume that the nuclear density is a spherically symmetric Schwartz function - as we do in the mathematical part of this paper - this implies that the vacuum polarization potential $U$ is bounded continuous and decreases exponentially at infinity.

However, if we assume that we have a point nucleus as assumed by Uehling 29], this is no longer the case. To relate to Uehling's work we will discuss this case as well although the corresponding potential is no longer form bounded with respect to the kinetic energy.

From (62) we have

$$
U=Z \sqrt{\frac{2}{\pi^{3}}} \mathcal{F}^{-1}\left(\frac{C}{|\cdot|^{4}}\right) .
$$

According to Uehling [29] and Schwinger [25], Equation (2.53), this is

$$
U(\mathbf{x})=\frac{2}{3 \pi} \frac{Z}{|\mathbf{x}|} \int_{1}^{\infty} e^{-2|\mathbf{x}| s}\left(1+\frac{1}{2 s^{2}}\right) \frac{\left(s^{2}-1\right)^{1 / 2}}{s^{2}} d s,
$$

which means asymptotically

$$
U(\mathbf{x})= \begin{cases}-\frac{2}{3 \pi} Z|\mathbf{x}|^{-1}\left(\log |\mathbf{x}|+\frac{5}{6}+\gamma\right)+O(1) & |\mathbf{x}| \rightarrow 0 \\ \frac{Z}{4 \sqrt{\pi}} e^{-2 \mid \mathbf{x}}|\mathbf{x}|^{-5 / 2}(1+O(1 /|\mathbf{x}|)) & |\mathbf{x}| \rightarrow \infty\end{cases}
$$

where $\gamma \approx 0.5772$ is Euler's constant. Consequently one obtains an effective potential

$$
\varphi_{\mathrm{eff}}(\mathbf{x})=-\alpha \varphi-\alpha^{2} U=-\alpha \frac{Z}{|\mathbf{x}|}+\alpha^{2} \frac{2}{3 \pi} \frac{Z}{|\mathbf{x}|}\left(\log |\mathbf{x}|+\frac{5}{6}+\gamma\right)+O(1)
$$

close to the nucleus.

Obviously, due to vacuum polarization, the effective potential becomes more singular than the Coulomb potential. This implies that the energy

$$
|\mathbf{p}|-\alpha^{2} \frac{2 Z}{3 \pi|\mathbf{x}|} \log \frac{1}{|\mathbf{x}|}
$$

is unbounded from below for all positive values of $\alpha$ and $Z$ and (66) is no longer relatively form bounded with respect to the relativistic kinetic energy operator. This suggests to avoid the mathematical idealization of a point nucleus and to take the experimental fact that the nuclei are extended into account.

3.6. Splitting of the Bound State Energies. The effect of the vacuum polarization potential to lowest order in $\alpha$ is given by the effective one-particle operator

$$
P_{+}^{\varphi}\left(D^{\varphi}-\alpha^{2} U(\mathbf{x})\right) P_{+}^{\varphi} .
$$

Therefore, the energy eigenvalues are shifted in lowest order of $\alpha$ by

$$
\delta E=-\alpha^{2} \int_{\mathbb{R}^{3}} d \mathbf{x} U(\mathbf{x})|\psi(\mathbf{x})|^{2}
$$

where $\psi(x)$ denotes an eigenstate of $D^{\varphi}$.

To get a rough heuristic estimate on the numerical effect Uehling [29] assumes the nucleus to be a point particle, i.e., its density is $n(\mathbf{x})=Z \delta(\mathbf{x})$, and takes 
the corresponding Schrödinger eigenstates $\psi_{n, l}$ where $n$ is the principal quantum number and $l$ the orbital-angular-momentum quantum number:

$$
\delta E_{n, l}=-\alpha^{2} \int_{\mathbb{R}^{3}} d \mathbf{x} U(\mathbf{x})\left|\psi_{n, l}(\mathbf{x})\right|^{2} \approx-\frac{4 Z \alpha^{2}}{15}\left|\psi_{n, l}(0)\right|^{2}=-\frac{4 Z^{4} \alpha^{5}}{15 \pi n^{3}} \delta_{0, l} .
$$

Concerning the first excited eigenvalue $n=2$ this indicates an energy level splitting (of the $2 s$ and $2 p$ state) of

$$
\delta E_{2,0} \sim-\frac{Z^{4} \alpha^{5} m}{30}
$$

the Uehling effect [29, Equation (29).

The vacuum polarization (69) accounts for only one percent of the $2 s_{1 / 2}-2 p_{1 / 2}$ Lamb shift of hydrogen, since the Bohr radius is much bigger than the range of the vacuum polarization potential. However, the Bohr radii of muonic atoms are much smaller because of the large effective mass which means that the vacuum polarization of muonic helium accounts for 90 percent of the Lamb shift (Peterman and Yamaguchi [21], Glauber et al. [9], see also Greiner et al. [10], p. 413).

\section{Proof of the Self-Adjointness of the Renormalized Hamiltonian}

In the following we are going to show that the quadratic form associated to the operator $\mathbb{H}$ of the electron-positron field restricted to the one-electron sector $\mathfrak{H}_{+}^{\varphi}$ defines a self-adjoint operator which is bounded from below. This means - among other things - that higher order renormalizations, as introduced in perturbation theory by Dyson 4, are unnecessary.

We will show infinitesimal form boundedness of all perturbations, namely $U, P_{3}$, and $P_{4}$ relative to $P_{+}^{\varphi} D^{\varphi} P_{+}^{\varphi}$. For $X$ this has been already shown in Lemma 2

$\mathbf{U}$ : According to (62) and the remark thereafter $U$ is bounded and therefore trivially infinitesimally relatively form bounded.

$\mathbf{P}_{4}$ : We will show that for any positive $\epsilon$ there exists a $C_{\epsilon}$ such that for all $\psi \in \mathcal{D}\left(D^{\varphi}\right) \subset \mathrm{L}^{3}(\Gamma) \cap \mathrm{L}^{2}(\Gamma)$

$$
\left(\psi, P_{4} \psi\right) \leq \epsilon\|\psi\|_{3}^{2}+C_{\epsilon}\|\psi\|_{2}
$$

which implies the infinitesimal form boundedness by the Sobolev's inequality for $\sqrt{-\Delta}$.

Lemma 3. Assume $\alpha Z \in[0,1), \chi \in L^{5}(\Gamma)$. Then

$$
\left|\operatorname{tr}\left(\chi Q_{4}\right)\right| \leq\left\|\chi Q_{4}\right\|_{1} \leq \frac{C_{\varphi, 4}}{3 \pi^{2}}\|\varphi\|_{5}^{4}\|\chi\|_{5}
$$

with

$$
C_{\varphi, 4}:=1+\alpha\left\|\varphi \frac{1}{D^{\varphi}}\right\|
$$

Proof. We have

$$
\begin{aligned}
\left|\operatorname{tr}\left(\chi Q_{4}\right)\right| & \leq \frac{1}{2 \pi} \int_{-\infty}^{\infty} d \eta\left\|\chi \frac{1}{D^{0}+i \eta} \varphi \frac{1}{D^{0}+i \eta} \varphi \frac{1}{D^{\varphi}+i \eta} \varphi \frac{1}{D^{0}+i \eta} \varphi \frac{1}{D^{0}+i \eta}\right\|_{1} \\
& \leq \frac{1}{2 \pi} \int_{-\infty}^{\infty} d \eta\left\|\chi \frac{1}{D^{0}+i \eta}\right\|_{5}\left\|\varphi \frac{1}{D^{0}+i \eta}\right\|_{5}^{3}\left\|\varphi \frac{1}{D^{\varphi}+i \eta}\right\|_{5} .
\end{aligned}
$$


(We use the standard notation $\|A\|_{p}=\sqrt[p]{\operatorname{tr}|A|^{p}}$.) We will estimate the right hand side of the above inequality which will also show that $\chi Q^{(4)} \in \mathfrak{S}_{1}(\mathfrak{H})$. To this end we estimate the factor containing the perturbed resolvent:

$$
\begin{aligned}
\left\|\varphi \frac{1}{D^{\varphi}+i \eta}\right\|_{5}=\| \varphi \frac{1}{D^{0}+i \eta}\left(D^{0}+i \eta\right) & \frac{1}{D^{\varphi}+i \eta} \|_{5} \\
& \leq\left\|\left(D^{0}+i \eta\right) \frac{1}{D^{\varphi}+i \eta}\right\|_{\infty}\left\|\varphi \frac{1}{D^{0}+i \eta}\right\|_{5} .
\end{aligned}
$$

The first factor on the right side is finite:

$$
\left\|\left(D^{0}+i \eta\right) \frac{1}{D^{\varphi}+i \eta}\right\|_{\infty} \leq 1+\alpha\left\|\varphi \frac{1}{D^{\varphi}+i \eta}\right\|_{\infty} \leq 1+\alpha\left\|\varphi \frac{1}{D^{\varphi}}\right\|_{\infty}<\infty
$$

where we use that $D^{\varphi}$ is invertible because of Lemma 1 and that $\varphi$ is bounded (see (4)). (Note the boundedness would also hold if $\varphi=Z \alpha /|\cdot|$, since $1 /|\cdot|$ is relatively bounded with respect to $\sqrt{-\Delta}$.)

Since

$$
\|f(x) g(-i \nabla)\|_{5} \leq(2 \pi)^{-3 / 5}\|f\|_{5}\|g\|_{5},
$$

the norm being the one of the trace ideal $\mathfrak{S}_{5}\left(L^{2}\left(\mathbb{R}^{3}\right)\right.$ ) (Simon [27], Theorem 4.1), we can estimate the other factors occurring in (73):

$$
\left\|\chi \frac{1}{D^{0}+i \eta}\right\|_{5} \leq \frac{1}{2^{1 / 5} \pi^{3 / 5}}\|\chi\|_{5}\left\|1 / \sqrt{|\cdot|^{2}+1+\eta^{2}}\right\|_{5}
$$

(norm in $\mathfrak{S}_{5}(\mathfrak{H})$ on the left hand side and in $\mathfrak{S}_{5}\left(L^{2}\left(\mathbb{R}^{3}\right)\right)$ on the right hand side) and a similar expression of the term containing $\varphi$.

Using (74) and (76) allows us to continue (173) as

$$
\begin{aligned}
\mid \operatorname{tr}\left(\chi Q_{4} \mid\right. & \leq \frac{C_{\varphi, 4}}{4 \pi^{4}}\|\chi\|_{5}\|\varphi\|_{5}^{4} \int_{-\infty}^{\infty} d \eta \int_{\mathbb{R}^{3}} d \mathbf{p} \frac{1}{\left(\mathbf{p}^{2}+1+\eta^{2}\right)^{5 / 2}} \\
& \leq \frac{C_{\varphi, 4}}{4 \pi^{4}} \int_{-\infty}^{\infty} d \eta \frac{1}{\left(\eta^{2}+1\right)} \int_{\mathbb{R}^{3}} \frac{1}{\left(1+\mathbf{p}^{2}\right)^{2}} d \mathbf{p}\|\chi\|_{5}\|\varphi\|_{5}^{4} \leq \frac{C_{\varphi, 4}}{3 \pi^{2}}\|\chi\|_{5}\|\varphi\|_{5}^{4} .
\end{aligned}
$$

This has the

Corollary 2. The perturbation $P_{4}$ is relatively form bounded with respect to $\left|D^{0}\right|$ with form bound zero.

Proof. We pick $\chi=|\psi|^{2} *|\cdot|^{-1}$ in Lemma 3 with $\psi \in H^{1 / 2}(\Gamma)$. Using Young's inequality followed by Sobolev's inequality yields the desired result.

$\mathbf{P}_{3}$ : Unfortunately, Simon's elegant trace inequality used in (73) does not suffice to handle the $Q_{3}$ containing only four resolvents. In that case we estimate directly:

Lemma 4. Denote the electric potential of a state $\psi \in H^{1 / 2}(\Gamma)$ of finite kinetic energy by $\chi(\mathbf{x}):=\int d y|\psi(y)|^{2} /|\mathbf{x}-\mathbf{y}|$ and assume $p>3$. Then there exists a constant $C_{\varphi, p}$ such that

$$
\left|\left(\psi, P_{3} \psi\right)\right| \leq C_{\varphi, p}\|\chi\|_{p} .
$$


Proof. We have

$$
\begin{aligned}
\left(\psi, P_{3} \psi\right)=\int_{\mathbb{R}^{3}} d \mathbf{x} \chi(\mathbf{x}) \rho_{3}(\mathbf{x})=\int_{\mathbb{R}^{3}} d \mathbf{p} \hat{\chi}(\mathbf{p}) \widehat{\rho_{3}}(\mathbf{p}) \\
\quad=(2 \pi)^{-3 / 2} \int_{\mathbb{R}^{3}} d \mathbf{p}_{1} \int_{\mathbb{R}^{3}} d \mathbf{p}_{2} \sum_{\sigma=1}^{4} \hat{\chi}\left(\mathbf{p}_{1}-\mathbf{p}_{2}\right) \hat{Q}_{3}\left(\mathbf{p}_{1}, \sigma ; \mathbf{p}_{2}, \sigma\right)
\end{aligned}
$$

where we use the Definition (17) of $\rho_{3}$. The "eigenfunctions" of the free Dirac operator in momentum space are

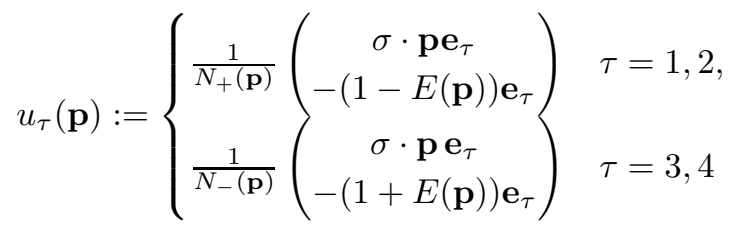

with $\mathbf{e}_{\tau}:=(1,0)^{t}$ for $\tau=1,3$ and $\mathbf{e}_{\tau}:=(0,1)^{t}$ for $\tau=2,4$ and

$$
N_{+}(\mathbf{p})=\sqrt{2 E(\mathbf{p})(E(\mathbf{p})-1)}, \quad N_{-}(\mathbf{p})=\sqrt{2 E(\mathbf{p})(E(\mathbf{p})+1)} .
$$

The indices 1 and 2 refer to positive "eigenvalue" $E(\mathbf{p})$ and the indices 3 and 4 to negative $-E(\mathbf{p})$. (See, e.g., Evans et al. 6].) Using Plancherel's theorem we get

$$
\begin{aligned}
& \left(\psi, P_{3} \psi\right)=\frac{1}{(2 \pi)^{7}} \int_{\mathbb{R}^{3}} d \mathbf{p}_{1} \int_{\mathbb{R}^{3}} d \mathbf{p}_{2} \int_{\mathbb{R}^{3}} d \mathbf{p}_{3} \int_{\mathbb{R}^{3}} d \mathbf{p}_{4} \sum_{\tau_{1}, \tau_{2}, \tau_{3}, \tau_{4}=1}^{4} \\
& \times\left\langle u_{\tau_{1}}\left(\mathbf{p}_{1}\right) \mid u_{\tau_{2}}\left(\mathbf{p}_{2}\right)\right\rangle\left\langle u_{\tau_{2}}\left(\mathbf{p}_{2}\right) \mid u_{\tau_{3}}\left(\mathbf{p}_{3}\right)\right\rangle\left\langle u_{\tau_{3}}\left(\mathbf{p}_{3}\right) \mid u_{\tau_{4}}\left(\mathbf{p}_{4}\right)\right\rangle\left\langle u_{\tau_{4}}\left(\mathbf{p}_{4}\right) \mid u_{\tau_{1}}\left(\mathbf{p}_{1}\right)\right\rangle \\
& \times \int_{-\infty}^{\infty} d \eta \frac{1}{\left(i a_{\tau_{1}} E\left(\mathbf{p}_{1}\right)-\eta\right)\left(i a_{\tau_{2}} E\left(\mathbf{p}_{2}\right)-\eta\right)\left(i a_{\tau_{3}} E\left(\mathbf{p}_{3}\right)-\eta\right)\left(i a_{\tau_{4}} E\left(\mathbf{p}_{4}\right)-\eta\right)},
\end{aligned}
$$

with $a_{\tau}=1$ for $\tau=1,2$ and $a_{\tau}=-1$ for $\tau=3,4$. The integral over $\eta$ is seen to vanish by Cauchy's theorem, if all four $a_{\tau_{j}}$ have the same sign. In fact we have to distinguish only two cases, namely three of the $a_{\tau_{j}}$ are equal and two of the $a_{\tau_{j}}$ are equal.

Therefore in the following we will only treat two different cases. The others then work analogously.

We begin with

$$
a_{\tau_{1}}=-1, a_{\tau_{2}}=a_{\tau_{3}}=a_{\tau_{3}}=1 .
$$

In that case the first factor in (81) reads

$$
\begin{aligned}
& \text { (83) } \sum_{\tau_{1}=3,4}\left\langle u_{\tau_{1}}\left(\mathbf{p}_{1}\right) \mid u_{\tau_{2}}\left(\mathbf{p}_{2}\right)\right\rangle \sum_{\tau_{2}=1,2}\left\langle u_{\tau_{2}}\left(\mathbf{p}_{2}\right) \mid u_{\tau_{3}}\left(\mathbf{p}_{3}\right)\right\rangle \times \\
& \times \sum_{\tau_{3}=1,2}\left\langle u_{\tau_{3}}\left(\mathbf{p}_{3}\right) \mid u_{\tau_{4}}\left(\mathbf{p}_{4}\right)\right\rangle \sum_{\tau_{4}=1,2}\left\langle u_{\tau_{4}}\left(\mathbf{p}_{4}\right) \mid u_{\tau_{1}}\left(\mathbf{p}_{1}\right)\right\rangle= \\
& \operatorname{tr}_{\mathbb{C}^{2}}\left[\frac{\sigma \cdot \mathbf{p}_{1} \sigma \cdot \mathbf{p}_{2}+\left(1+E\left(\mathbf{p}_{1}\right)\right)\left(1-E\left(\mathbf{p}_{2}\right)\right)}{N_{-}\left(\mathbf{p}_{1}\right)^{2} N_{+}\left(\mathbf{p}_{2}\right)^{2} N_{+}\left(\mathbf{p}_{3}\right)^{2} N_{+}\left(\mathbf{p}_{4}\right)^{2}}\left[\sigma \cdot \mathbf{p}_{2} \sigma \cdot \mathbf{p}_{3}+\left(1-E\left(\mathbf{p}_{2}\right)\right)\left(1-E\left(\mathbf{p}_{3}\right)\right)\right]\right. \\
& \left.\times\left[\sigma \cdot \mathbf{p}_{3} \sigma \cdot \mathbf{p}_{4}+\left(1-E\left(\mathbf{p}_{3}\right)\right)\left(1-E\left(\mathbf{p}_{4}\right)\right)\right]\left[\sigma \cdot \mathbf{p}_{4} \sigma \cdot \mathbf{p}_{1}+\left(1-E\left(\mathbf{p}_{4}\right)\right)\left(1+E\left(\mathbf{p}_{1}\right)\right)\right]\right] .
\end{aligned}
$$


We estimate the modulus of (83) and obtain

$$
\begin{aligned}
|\underline{831}| & \leq \mathrm{c} \frac{\operatorname{tr}_{\mathbb{C}^{2}}\left|\sigma \cdot \mathbf{p}_{4} \sigma \cdot \mathbf{p}_{1}+\left(1-E\left(\mathbf{p}_{4}\right)\right)\left(1+E\left(\mathbf{p}_{1}\right)\right)\right|}{N_{-}\left(\mathbf{p}_{1}\right) N_{+}\left(\mathbf{p}_{4}\right)} \\
& \leq \mathrm{c} \frac{\left|\mathbf{p}_{4} \cdot \mathbf{p}_{1}-\left(E\left(\mathbf{p}_{4}\right)-1\right)\left(1+E\left(\mathbf{p}_{1}\right)\right)\right|+\left|\mathbf{p}_{4} \wedge \mathbf{p}_{1}\right|}{N_{-}\left(\mathbf{p}_{1}\right) N_{+}\left(\mathbf{p}_{4}\right)}
\end{aligned}
$$

(Here and in the following $\mathrm{c}$ is a generic positive constant.) Since

$$
\begin{aligned}
\frac{1}{2 \pi} \int_{-\infty}^{\infty} d \eta \frac{1}{\left(-i E\left(\mathbf{p}_{1}\right)-\eta\right)\left(i E\left(\mathbf{p}_{2}\right)-\eta\right)\left(i E\left(\mathbf{p}_{3}\right)-\eta\right)\left(i E\left(\mathbf{p}_{4}\right)-\eta\right)} \\
=\frac{1}{\left(E\left(\mathbf{p}_{2}\right)+E\left(\mathbf{p}_{3}\right)\right)\left(E\left(\mathbf{p}_{2}\right)+E\left(\mathbf{p}_{3}\right)\right)\left(E\left(\mathbf{p}_{3}\right)+E\left(\mathbf{p}_{4}\right)\right)}
\end{aligned}
$$

our term of interest (81) is bounded by constant times

$$
\begin{aligned}
\int_{\mathbb{R}^{3}} d \mathbf{p}_{1} \int_{\mathbb{R}^{3}} d \mathbf{p}_{2} \int_{\mathbb{R}^{3}} d \mathbf{p}_{3} \int_{\mathbb{R}^{3}} d \mathbf{p}_{4}\left|\hat{\chi}\left(\mathbf{p}_{1}-\mathbf{p}_{2}\right) \hat{\varphi}\left(\mathbf{p}_{2}-\mathbf{p}_{3}\right) \hat{\varphi}\left(\mathbf{p}_{3}-\mathbf{p}_{4}\right) \hat{\varphi}\left(\mathbf{p}_{4}-\mathbf{p}_{1}\right)\right| \\
\left.\quad \times \frac{\left|\mathbf{p}_{4} \cdot \mathbf{p}_{1}-\left(E\left(\mathbf{p}_{4}\right)-1\right)\left(E\left(\mathbf{p}_{1}\right)+1\right)\right|+\left|\mathbf{p}_{4} \wedge \mathbf{p}_{1}\right|}{N_{-}\left(\mathbf{p}_{1}\right) N_{+}\left(\mathbf{p}_{4}\right)\left(E\left(\mathbf{p}_{2}\right)+E\left(\mathbf{p}_{3}\right)\right)\left(E\left(\mathbf{p}_{2}\right)+E\left(\mathbf{p}_{3}\right)\right)\left(E\left(\mathbf{p}_{3}\right)+E\left(\mathbf{p}_{4}\right)\right.}\right) .
\end{aligned}
$$

Substituting $\mathbf{p}_{2} \rightarrow \mathbf{p}_{1}+\mathbf{p}_{2}$ turns (84) into

$$
\begin{aligned}
& \int_{\mathbb{R}^{3}} d \mathbf{p}_{1} \int_{\mathbb{R}^{3}} d \mathbf{p}_{2} \int_{\mathbb{R}^{3}} d \mathbf{p}_{3} \int_{\mathbb{R}^{3}} d \mathbf{p}_{4} \\
& \left|\hat{\chi}\left(-\mathbf{p}_{2}\right)\right| \frac{\left|\hat{\varphi}\left(\mathbf{p}_{2}+\mathbf{p}_{1}-\mathbf{p}_{3}\right) \hat{\varphi}\left(\mathbf{p}_{3}-\mathbf{p}_{4}\right) \hat{\varphi}\left(\mathbf{p}_{4}-\mathbf{p}_{1}\right)\right|}{N_{-}\left(\mathbf{p}_{1}\right) N_{+}\left(\mathbf{p}_{4}\right)} \\
& \times \frac{\left|\mathbf{p}_{4} \cdot \mathbf{p}_{1}-\left(E\left(\mathbf{p}_{4}\right)-1\right)\left(E\left(\mathbf{p}_{1}\right)+1\right)\right|+\left|\mathbf{p}_{4} \wedge \mathbf{p}_{1}\right|}{\left(E\left(\mathbf{p}_{2}+\mathbf{p}_{1}\right)+E\left(\mathbf{p}_{3}\right)\right)\left(E\left(\mathbf{p}_{2}+\mathbf{p}_{1}\right)+E\left(\mathbf{p}_{3}\right)\right)\left(E\left(\mathbf{p}_{3}\right)+E\left(\mathbf{p}_{4}\right)\right.} \\
& =\int_{\mathbb{R}^{3}} d \mathbf{p}_{2}\left|\hat{\chi}\left(-\mathbf{p}_{2}\right)\right| f\left(\mathbf{p}_{2}\right)
\end{aligned}
$$

where we introduce $f$ to be the remaining integrand. We will now estimate $f$. Substituting $\mathbf{p}_{1} \rightarrow \mathbf{p}_{1}+\mathbf{p}_{4}, \mathbf{p}_{3} \rightarrow \mathbf{p}_{3}+\mathbf{p}_{4}$ we get

$$
\begin{aligned}
f\left(\mathbf{p}_{2}\right)= & \int_{\mathbb{R}^{3}} d \mathbf{p}_{1} \int_{\mathbb{R}^{3}} d \mathbf{p}_{3} \int_{\mathbb{R}^{3}} d \mathbf{p}_{4} \hat{\varphi}\left(\mathbf{p}_{2}+\mathbf{p}_{1}-\mathbf{p}_{3}\right) \hat{\varphi}\left(\mathbf{p}_{3}\right) \hat{\varphi}\left(\mathbf{p}_{1}\right) \mid \\
& \times \frac{\left|\mathbf{p}_{4} \cdot\left(\mathbf{p}_{1}+\mathbf{p}_{4}\right)-\left(E\left(\mathbf{p}_{4}\right)-1\right)\left(1+E\left(\mathbf{p}_{1}+\mathbf{p}_{4}\right)\right)\right|+\left|\mathbf{p}_{4} \wedge \mathbf{p}_{1}\right|}{N_{-}\left(\mathbf{p}_{1}+\mathbf{p}_{4}\right) N_{+}\left(\mathbf{p}_{4}\right)\left(E\left(\mathbf{p}_{2}+\mathbf{p}_{1}+\mathbf{p}_{4}\right)+E\left(\mathbf{p}_{3}+\mathbf{p}_{4}\right)\right)} \\
& \quad \times \frac{1}{\left(E\left(\mathbf{p}_{2}+\mathbf{p}_{1}+\mathbf{p}_{4}\right)+E\left(\mathbf{p}_{3}+\mathbf{p}_{4}\right)\right)\left(E\left(\mathbf{p}_{3}+\mathbf{p}_{4}\right)+E\left(\mathbf{p}_{4}\right)\right)} .
\end{aligned}
$$

Since

$$
E\left(\mathbf{p}_{1}+\mathbf{p}_{4}\right)=E\left(\mathbf{p}_{4}\right)+\frac{\mathbf{p}_{4} \cdot \mathbf{p}_{1}}{E\left(\mathbf{p}_{4}\right)} \mu
$$

for some $\mu \in[0,1]$, we see that

$$
\left|\mathbf{p}_{4} \cdot\left(\mathbf{p}_{1}+\mathbf{p}_{4}\right)-\left(E\left(\mathbf{p}_{4}\right)-1\right)\left(E\left(\mathbf{p}_{1}+\mathbf{p}_{4}\right)+1\right)\right|+\left|\mathbf{p}_{4} \wedge \mathbf{p}_{1}\right| \leq 4\left|\mathbf{p}_{1}\right|\left|\mathbf{p}_{4}\right| .
$$

Notice, we can bound

$$
\int_{\mathbb{R}^{3}} d \mathbf{p}_{4} \frac{\left|\mathbf{p}_{4}\right|}{E\left(\mathbf{p}_{3}+\mathbf{p}_{4}\right) E\left(\mathbf{p}_{4}\right)^{2} N_{+}\left(\mathbf{p}_{1}+\mathbf{p}_{4}\right)} \leq \mathrm{c}
$$


independent of $\mathbf{p}_{1}$ and $\mathbf{p}_{3}$. Therefore,

$$
f\left(\mathbf{p}_{2}\right) \leq \mathrm{c} \int_{\mathbb{R}^{3}} d \mathbf{p}_{1} \int_{\mathbb{R}^{3}} d \mathbf{p}_{3}\left|\hat{\varphi}\left(\mathbf{p}_{2}+\mathbf{p}_{1}-\mathbf{p}_{3}\right) \hat{\varphi}\left(\mathbf{p}_{3}\right) \hat{\varphi}\left(\mathbf{p}_{1}\right)\right|\left|\mathbf{p}_{1}\right| .
$$

Since $\hat{\varphi}(\mathbf{k})=4 \pi \hat{n}(\mathbf{k}) / \mathbf{k}^{2}$, we have that $f(0)$ is finite; since $\hat{n}$ is compactly supported, $\mathbf{p}_{1}$ and $\mathbf{p}_{2}$ are bounded. We conclude that $f$ has also compact support.

Consequently, since

$$
\int_{\mathbb{R}^{3}} d \mathbf{p}_{2}\left|\hat{\chi}\left(-\mathbf{p}_{2}\right)\right| f\left(\mathbf{p}_{2}\right) \leq\|\hat{\chi}\|_{q}\|f\|_{p}
$$

we see using the Hausdorff-Young inequality that for all $p \geq 2$

$$
\int_{\mathbb{R}^{3}} d \mathbf{p}_{2}\left|\chi\left(-\mathbf{p}_{2}\right)\right| f\left(\mathbf{p}_{2}\right) \leq \mathrm{c}_{p, \varphi}\|\chi\|_{p}
$$

for constant $\mathrm{c}_{p, \varphi}$ depending on $p$ and $\varphi$.

Next, we take a peek at the case $a_{\tau_{1}}=a_{\tau_{2}}=1$ and $a_{\tau_{3}}=a_{\tau_{4}}=-1$. The corresponding integral over $\eta$ gives

$$
\begin{aligned}
\frac{1}{2 \pi} \int_{-\infty}^{\infty} d \eta \frac{1}{\left(i E\left(\mathbf{p}_{1}\right)-\eta\right)\left(i E\left(\mathbf{p}_{2}\right)-\eta\right)\left(-i E\left(\mathbf{p}_{3}\right)-\eta\right)\left(-i E\left(\mathbf{p}_{4}\right)-\eta\right)} \\
=\frac{1}{\left(E\left(\mathbf{p}_{2}\right)+E\left(\mathbf{p}_{3}\right)\right)\left(E\left(\mathbf{p}_{2}\right)+E\left(\mathbf{p}_{4}\right)\right)\left(E\left(\mathbf{p}_{1}\right)+E\left(\mathbf{p}_{4}\right)\right)} \\
+\frac{1}{\left(E\left(\mathbf{p}_{2}\right)+E\left(\mathbf{p}_{3}\right)\right)\left(E\left(\mathbf{p}_{1}\right)+E\left(\mathbf{p}_{3}\right)\right)\left(E\left(\mathbf{p}_{1}\right)+E\left(\mathbf{p}_{4}\right)\right)} .
\end{aligned}
$$

Observe now that the corresponding first factor in 81] can be bounded by $\mathrm{c}$. $4 \frac{\left|\mathbf{p}_{2}\right|\left|\mathbf{p}_{3}\right|}{N_{+}\left(\mathbf{p}_{2}\right) N_{-}\left(\mathbf{p}_{3}\right)}$. Now, we do similar variable transforms as above and arrive at an analogue of (87).

Again this has the

Corollary 3. The perturbation $P_{3}$ is relatively form bounded with respect to $\left|D^{0}\right|$ with form bound zero.

The above result was the final step in showing that all four perturbations, $U$, $P_{3}, P_{4}$, and $X$, are form bounded with respect to $P_{+}^{\varphi} D^{\varphi} P_{+}^{\varphi}$.

\section{REFERENCES}

[1] H.A. Bethe. The electromagnetic shift of energy levels. Phys. Rev., Minneapolis, II. Ser., 72:339-341, 1947.

[2] P.-A.-M. Dirac. Théorie du positron. In Cockcroft, J. Chadwick, F. Joliot, J. Joliot, N. Bohr, G. Gamov, P.A.M. Dirac, and W. Heisenberg, editors, Structure et propriétés des noyaux atomiques. Rapports et discussions du septieme conseil de physique tenu à Bruxelles du 22 au 29 octobre 1933 sous les auspices de l'institut international de physique Solvay. Publies par la commission administrative de l'institut., pages 203-212. Paris: Gauthier-Villars. XXV, 353 S., 1934.

[3] P.A.M. Dirac. Discussion of the infinite distribution of electrons in the theory of the positron. Proc. Camb. Philos. Soc., 30:150-163, 1934.

[4] F. J. Dyson. The radiation theories of Tomonaga, Schwinger, and Feynman. Physical Rev. (2), 75:486-502, 1949.

[5] F. J. Dyson. Divergence of perturbation theory in quantum electrodynamics. Physical Rev. (2), 85:631-632, 1952. 
[6] William Desmond Evans, Peter Perry, and Heinz Siedentop. The spectrum of relativistic oneelectron atoms according to Bethe and Salpeter. Comm. Math. Phys., 178(3):733-746, July 1996.

[7] J.D. French and V.F. Weisskopf. The Electromagnetic Shift of Energy Levels. Phys. Rev., II. Ser., 75:1240-1248, 1949.

[8] W.H. Furry and J.R. Oppenheimer. On the Theory of the Electron and Positive. Phys. Rev., II. Ser., 45:245-262, 1934.

[9] Roy Glauber, William Rarita, and Philip Schwed. Vacuum polarization effects on energy levels in $\mu$-mesonic atoms. Physical Review, 120(2):609-613, October 1960.

[10] W. Greiner, B. Müller, and J. Rafelski. Quantum Electrodynamics of Strong Fields. Texts and Mongraphs in Physics. Springer-Verlag, Berin and Heidelberg and New York and Tokyo, 1 edition, 1985.

[11] W. Heisenberg. Bemerkungen zur Diracschen Theorie des Positrons. Z. Phys., 90:209-231, 1934.

[12] Bernard Helffer and Heinz Siedentop. Form perturbations of the second quantized Dirac field. Math. Phys. Electron. J., 4:Paper 4, 16 pp. (electronic), 1998.

[13] J. M. Jauch and F. Rohrlich. The theory of photons and electrons. The relativistic quantum field theory of charged particles with spin one-half. Addison-Wesley Publishing Company, Inc., Cambridge, Massachusetts, 1955.

[14] Tosio Kato. Perturbation Theory for Linear Operators, volume 132 of Grundlehren der mathematischen Wissenschaften. Springer-Verlag, Berlin, 1 edition, 1966.

[15] M. Klaus and G. Scharf. The regular external field problem in quantum electrodynamics. Helv. Phys. Acta, 50(6):779-802, 1977.

[16] M. Klaus and G. Scharf. Vacuum polarization in Fock space. Helv. Phys. Acta, 50(6):803-814, 1977.

[17] Norman M. Kroll and Willis E. Lamb jun. On the Self-Energy of a Bound Electron. Phys. Rev., II. Ser., 75:388-398, 1949.

[18] Willis E. Lamb and Robert C. Retherford. Fine structure of the hydrogen atom by a microwave method. Physical Review, 72(3):241-243, August 1947.

[19] Peter W. Milonni. The Quantum Vacuum: An Introduction to Quantum Electrodynamics. Academic Press, Inc., Boston, 1 edition, 1994.

[20] W. Pauli and M.E. Rose. Remarks on the Polarization Effects in the Positron Theory. Phys. Rev., II. Ser., 49:462-465, 1936.

[21] A. Peterman and Y. Yamaguchi. Corrections to the $3 \mathrm{~d}-2 \mathrm{p}$ transitions in $\mu$-mesonic phosphorus and the mass of the muon. Physical Review Letters, 2(8):359-361, April 1959.

[22] Michael Reed and Barry Simon. Methods of modern mathematical physics. II. Fourier analysis, self-adjointness. Academic Press [Harcourt Brace Jovanovich Publishers], New York, 1975.

[23] Michael Reed and Barry Simon. Methods of Modern Mathematical Physics, volume 4: Analysis of Operators. Academic Press, New York, 1 edition, 1978.

[24] Silvan S. Schweber. QED and the men who made it: Dyson, Feynman, Schwinger, and Tomonaga. Princeton Series in Physics. Princeton University Press, Princeton, NJ, 1994.

[25] Julian Schwinger. Quantum Electrodynamics II. Vacuum Polarization and Self-Energy. Phys. Rev., II. Ser., 75:651-679, 1949.

[26] Robert Serber. Linear modifications in the Maxwell field equations. Phys. Rev., II. Ser., 48:49-54, 1935.

[27] Barry Simon. Trace Ideals and their Applications, volume 35 of London Mathematical Society Lecture Note Series. Cambridge University Press, Cambridge, 1979.

[28] Bernd Thaller. The Dirac Equation. Texts and Monographs in Physics. Springer-Verlag, Berlin, 1 edition, 1992.

[29] E.A. Uehling. Polarization effects in the positron theory. Phys. Rev., II. Ser., 48:55-63, 1935.

[30] Steven Weinberg. The quantum theory of fields. Vol. I. Cambridge University Press, Cambridge, 1996. Foundations, Corrected reprint of the 1995 original.

[31] V. Weisskopf. Über die Elektrodynamik des Vakuums auf Grund der Quantentheorie des Elektrons. Math.-Fys. Medd., Danske Vid. Selsk., 16(6):1-39, 1936. 
Mathematik, Theresienstrasse 39, 80333 München, Germany. Address as of March 1, 2003: Ceremade, Université Paris-Dauphine, Place du Maréchal de Lattre de TasSigny, F-75775 Paris Cedex 16, France.

E-mail address: hainzl@ceremade.dauphine.fr

Mathematik, Theresienstrasse 39, 80333 München, Germany.

E-mail address: h.s@lmu.de 